\title{
Review
}

\section{Gold nanoparticles in cancer therapy}

\author{
Zhao-Zhin Joanna LIM ${ }^{1}$, Jia-En Jasmine $\mathrm{LI}^{1,2}$, Cheng-Teng $\mathrm{NG}^{1,2}$, Lin-Yue Lanry YUNG ${ }^{2, *}$, Boon-Huat BAY1, * \\ ${ }^{1}$ Department of Anatomy, National University of Singapore, Singapore; ${ }^{2}$ Department of Chemical and Biomolecular Engineering, \\ National University of Singapore, Singapore
}

\begin{abstract}
The rapid advancement of nanotechnology in recent years has fuelled a burgeoning interest in the field of nanoparticle research, in particular, its application in the medical arena. A constantly expanding knowledge based on a better understanding of the properties of gold nanoparticles (AuNPs) coupled with relentless experimentation means that the frontiers of nanotechnology are constantly being challenged. At present, there seems to be heightened interest in the application of AuNPs to the management of cancer, encompassing diagnosis, monitoring and treatment of the disease. These efforts are undertaken in the hope of revolutionizing current methods of treatment and treatment strategies for a multifactorial disease such as cancer. This review will focus on the current applications of AuNPs in cancer management.
\end{abstract}

Keywords: gold nanoparticles; cancer therapeutics; cancer diagnosis; photothermal therapy; radiation therapy

Acta Pharmacologica Sinica (2011) 32: 983-990; doi: 10.1038/aps.2011.82; published online 11 Jul 2011

\section{Introduction}

According to the World Health Organization (WHO), cancer accounted for 7.9 million deaths in 2007 making it the leading cause of death in the world. Deaths from cancer around the globe are expected to climb upwards with an estimated 12 million deaths by cancer in $2030^{[1]}$. The frontiers of cancer research are therefore consistently challenged in order to advance the most effective means of cancer diagnosis, monitoring and treatment. Findings gleaned from cancer research would inevitably benefit mankind and save countless lives.

Current therapies employed for the treatment of cancer include surgery, chemotherapy and radiation therapy among others. While these methods have been accepted and practiced for decades, they have their drawbacks and side effects. Surgical removal of tumors is restricted mainly to large, resectable and accessible tumors. Chemotherapeutic drugs target rapidly dividing cells, and thus not only kill cancer cells, but also destroy normal cells like bone marrow cells and immune cells $^{[2]}$. This gives rise to widespread "collateral damage" in the patient's body. Radiation therapy involves the use of highenergy radiation like $X$-rays and gamma rays to destroy tumor cells, and inevitably causes deleterious effects to healthy tissues along the radiation path $^{[3]}$.

\footnotetext{
* To whom correspondence should be addressed.

E-mail antbaybh@nus.edu.sg (Boon-Huat BAY); cheyly@nus.edu.sg (Lin-Yue Lanry YUNG)

Received 2011-01-18 Accepted 2011-05-23
}

In light of the shortcomings of current treatment modalities for cancer, a critical thrust towards improving cancer therapy is to specifically target therapeutic agents to tumor cells while sparing healthy tissues from harm. This is one of the emerging interests in nanotechnology research. Nanotechnology refers to the manufacture of materials having nanoscale dimensions between $1 \mathrm{~nm}$ and $100 \mathrm{~nm}^{[4]}$. The small size of these nanomaterials confers their uniqueness with chemical and physical properties that are distinct from their bulk materials ${ }^{[5]}$. The rapid expansion in nanomaterial research increases the future prospect of novel diagnostic methods and treatment of diseases that plague mankind. This branch of nanotechnology in disease diagnosis, monitoring and treatment has been termed "nanomedicine" by the National Institutes of Health in the $\mathrm{USA}^{[4]}$.

Among the many nanomaterials being developed for nanomedicine applications, this review will focus on gold nanoparticles (AuNPs) and their potential as tumor sensors, drug delivery agents and enhancers in plasmonic photothermal therapy for the eradication of cancers. The use of AuNPs is gaining popularity in these areas of research for several reasons. Firstly, AuNPs are considered to be relatively biologically non-reactive and therefore suitable for in vivo applications compared to the very toxic cadmium and silver NPs ${ }^{[6]}$ although various groups (as explained in the later sections) are challenging this view. Other advantageous qualities include the strong optical properties of AuNPs due to localized surface plasmon resonance (LSPR) ${ }^{[7]}$, easily controllable surface 
chemistry which enables versatility in adding surface functional groups ${ }^{[8]}$, and lastly, the ease in control over particle size and shape during synthesis ${ }^{[9]}$. AuNPs may be considered to be fully multifunctional, with the possibility of combining different desired functionalities in one molecular-sized package. All these factors contribute to the strong interest and preference for the use of AuNPs over other NPs ${ }^{[10]}$. Examples of other nanomaterials for biomedical applications can be found in other published papers which expound on the utilization of quantum dots ${ }^{[11,12]}$, functionalized fullerene-based nanomateri$\mathrm{als}^{[13]}$ and magnetic NPs ${ }^{[14,15]}$ for the diagnosis and treatment of human diseases.

\section{AuNPs and cytotoxicity}

As the utility of AuNPs largely depends on the degree of inherent toxicity, studies on the toxicological profile of these NPs are discussed proceeding to their usage in cancer management. Since NPs exhibit properties which are markedly different from that of their much larger counterparts, their behaviour and effects cannot be extrapolated from information derived from their bulk materials. Bulk gold has generally been considered an inert metal valued for medicinal purposes ${ }^{[16]}$ and AuNPs have been thought to be likewise. In the literature, AuNPs have been reported to lack the ability to induce adverse and acute toxicity ${ }^{[17]}$ and are thus deemed to be biocompatible entities for use in biomedical applications ${ }^{[17,18]}$. However, recent studies have shown that there could be more to AuNP toxicity than already surmised and that the extent of toxicity response is closely associated with the size of the $\mathrm{AuNPS}^{[19,20]}$. Investigations have revealed that decreasing the size of NPs correlated with more widespread tissue distribution, heightened potential to deeper penetration within certain tissues, more effective internalization by cells, and increased toxic effects ${ }^{[21]}$. In terms of surface functionality, studies have shown that modification of the AuNP surface affect its uptake, interactions with cellular constituents and cytotoxicity ${ }^{[22,23]}$.

\section{In vitro studies on cytotoxicity of AuNPs}

Multiple studies have shown that AuNPs exert their cytotoxicity through the induction of oxidative stress. For example, when exposed to $1.4 \mathrm{~nm}$ AuNPs, HeLa cervical carcinoma cells exhibited increased reactive oxygen species (ROS) production and oxidative stress, leading to protein and lipid oxidation, severely impaired mitochondrial function, and eventually cell death ${ }^{[23]}$. The same investigators showed that Z-VAD-fmk, a caspase inhibitor was unable to rescue the cells from dying, leading to the conclusion that cells were killed by necrosis. Furthermore, genome-wide mRNA expression analysis verified that treatment with AuNPs caused up-regulation of stress-related and inflammation-related genes and a concomitant decrease in the expression of cell cycle genes. It appears that continual production of endogenous ROS within the cell exhausted the intracellular antioxidant pool and therefore induced irreversible damage that eventually lead to necrosis.

Oxidative stress was observed in MRC-5 fetal human lung fibroblast cells following exposure to $20 \mathrm{~nm} \mathrm{AuNPs}{ }^{[24]}$ with concomitant down-regulation of cell cycle genes such as Cyclin B2 and B1 and DNA damage response genes. In a follow-up study, the same investigators observed the presence of autophagy (validated by biochemical and morphological parameters) concurrent with oxidative stress in the lung fibroblasts following uptake of AuNPs ${ }^{[25]}$. It was also demonstrated in the same study that AuNP treatment led to the up-regulation of antioxidants and expression of stress-response genes and proteins, lending support to the hypothesis that oxidative stress could be a manifestation of AuNP cytotoxicity.

\section{In vivo studies on cytotoxicity of AuNPs}

In a recent study, blue mussel Mytilus edulis was observed to experience oxidative stress within $24 \mathrm{~h}$ of exposure to AuNPs ${ }^{[26]}$, indicating the possible impact of AuNPs to the ecosystem and aquatic animals. The same investigators also proposed the use of $M$ edulis as an ideal animal model for environmental toxicology studies of NPs. Another in vivo study utilized zebrafish embryos to assess the feasibility of AuNPs as probes for embryonic imaging ${ }^{[27]}$. In this study, the real-time effects of AuNPs on zebrafish embryos were investigated, and results showed that owing to the random diffusion of AuNPs to various parts of the embryo, toxic effects influencing the developmental outcome of the embryo were largely stochastic in nature. Among the $76 \%$ of zebrafish embryos that survived, only a minority $(2 \%)$ of zebrafish embryos exhibited deformities while the remaining $74 \%$ developed normally. The authors therefore proposed that given its relatively non-toxic nature, AuNPs could be exploited for in vivo imaging applications for embryonic studies.

For mammals, however, there is at present limited information regarding the in vivo toxicity of AuNPs. Studies have largely focused on the biodistribution of AuNPs in the body. A rat model study revealed the size-dependent organ distribution of AuNPs following intravenous (iv) administration. For $10 \mathrm{~nm}$ AuNPs, the distribution was found to be widespread, permeating the blood and organs of the cardio-respiratory system, immune system (such as spleen and thymus) and reproductive system, liver, kidney, and brain, whereas larger AuNPs (50, 100, and $250 \mathrm{~nm}$ ) were localized only to the blood, liver and spleen $^{[28]}$. A similar study conducted using 15, 50, 100, and $200 \mathrm{~nm}$ AuNPs showed that while the AuNPs with the largest dimension could only accumulate minimally in organs following iv administration into mice, AuNPs with the smallest dimension were detected in all tissues including blood and other organs such as the liver, lung, spleen, kidney, brain, stomach, and heart ${ }^{[29]}$. The results imply that smaller size AuNPs are more accessible to various tissues in the body and therefore the propensity to cause widespread harm, if any.

Another group of researchers assessed the in vivo toxicity of $13 \mathrm{~nm}$ AuNPs coated with poly (ethylene) glycol (PEG) in mice and showed that following iv injection of AuNPs, the NPs accumulated in mouse liver and spleen for up to a week, and induced acute inflammation and apoptosis in the liver ${ }^{[30]}$. The same group of investigators also demonstrated that iv administration of $4 \mathrm{~nm}$ or $100 \mathrm{~nm}$ PEG-coated AuNPs in mice 
induced up-regulation of common genes associated with apoptosis, cell cycle, inflammation, and metabolic process in liver tissues ${ }^{[31]}$.

A major challenge in the field of investigating in vivo cytotoxicity of NPs is the plausibility of translating observed cellular and immunological toxicity in animal models to humans, since there are distinct intra- and interspecies variations which need to be considered.

\section{Applications of AuNPs in cancer management} AuNPs as sensors for probing and imaging tumor cells

AuNPs are good candidates for labelling applications because of their ability to interact strongly with visible light. Upon exposure to light, free electrons in gold atoms are excited to a state of collective oscillation known as surface plasmon resonance (SPR), conferring gold the ability to absorb and scatter visible light ${ }^{[32]}$. In labelling applications, AuNPs are targeted and accumulated at the site of interest and based on their optical scattering properties, they enable visualization of the region under study. AuNPs may then be detected by any of the following ways: phase contrast optical microscopy, dark field microscopy, photothermal imaging, and photoacoustic imaging ${ }^{[33]}$. In addition, owing to its high atomic weight, AuNPs remain the preferred label for visualization and immuno-staining at the ultrastructural level using transmission electron microscopy ${ }^{[34]}$.

A crucial step in successful cancer therapy involves early diagnosis. The strong optical scattering properties of AuNPs, coupled with their relative biocompatibility, make them suitable as probes for cancer imaging. Through the conjugation of antibodies specific for antigens overexpressed on tumor cells, AuNPs can be directed to tumor cells, thus pin pointing their precise location in the body (Figure 1). It has been demonstrated that antibody-conjugated hollow gold nanospheres can be used for the surface-enhanced Raman

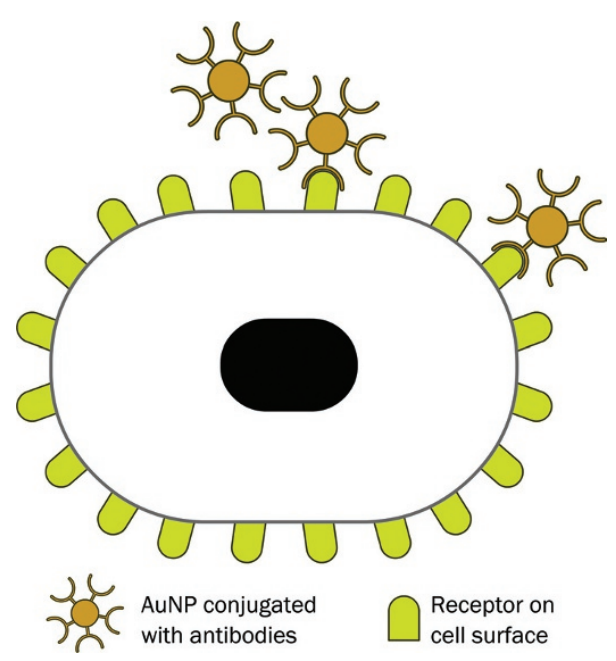

Figure 1. Schematic diagram showing the localization of antibody conjugated gold to receptors present on the plasma membrane of cells. spectroscopy (SERS) imaging of tumor biomarkers which are overexpressed in MCF7 breast cancer cells ${ }^{[35]}$. Raman scattering is a phenomenon that results from the inelastic collision of photons with molecules where energy, which is either lost or gained, translates to a change in the frequency of the scattered photons. This unique shift of frequency depends on the characteristic energy of molecular vibrations constituting the signal, hence a Raman spectrum consisting of different signals from molecular vibrations forms a "vibrational fingerprint" of a molecule ${ }^{[36]}$. In SERS, these Raman signals are amplified several folds by nanostructures present in the vicinity of the molecules. Gold and silver have been shown to cause significant enhancement ${ }^{[37]}$ and are thus the favoured nanostructures used as sensors ${ }^{[24]}$. By attaching a reporter to SERS sensors, targeted sensitive probing of molecules or structures within cells may be achieved ${ }^{[36]}$.

Recent studies have demonstrated the potential use of AuNPs for in vivo targeted imaging of cancer using Raman spectroscopy. Large optical enhancements can possibly be achieved in the detection of tumors in live animals owing to the 14-15 orders of magnitude signal amplification by AuNPs $^{[38]}$. Following the conjugation of AuNPs with appropriate ligands, cancer markers such as epidermal growth factor receptors present on the surface of human cancer cells and in xenograft tumor models could be targeted for detection. This shows the potential of using AuNPs for biomedical imaging in live subjects. However, it is important to note that the successful optical imaging performed in mice cannot be directly scaled up for in vivo imaging of human subjects because the optical signals possess limited tissue penetration ability ${ }^{[39]}$. At present, optical imaging only appears useful for tissues close to the skin surface or accessible by endoscopy. Hence, additional technological improvements are needed before Raman scattering by AuNPs can be used in a clinical setting.

The utility of AuNPs as novel biosensors for the detection of tumor cells can be demonstrated through the use of a screenprinted carbon electrode (SPCE) coupled with a NP-based electrocatalytic method ${ }^{[40]}$. Using this technique, in situ tumor cell proliferation was detected and quantified via the reaction of cell surface proteins with specific antibodies conjugated to AuNPs. Human tumor HMy2 cells (human leukocyte antigen (HLA-DR) class II positive B cells) and human tumor PC-3 cells (HLA-DR class II negative prostrate carcinoma) were first grown on the surface of SPCEs, following which they were incubated with a commercial monoclonal antibody (mAb) specific to DR molecules conjugated to AuNPs (direct method) or unconjugated $\mathrm{mAb}$ followed by secondary antibodies conjugated to AuNPs (indirect method). When hydrogen ions were catalytically reduced to hydrogen in the presence of AuNPs, the amount of AuNPs (and thus a corresponding indication of the quantity of attached tumor cells) could be quantified. In both methods, the AuNP immunosensor was able to distinguish DR-positive tumor cells from DR-negative tumor cells, showing the efficiency of this novel biosensor in detecting specific tumor cells. 


\section{AuNPs as drug delivery agents targeted to cancer cells}

A prominent application of AuNPs is their use as vehicles for delivery of molecules into cells. AuNPs have been described as "promising nanocarriers for therapeutics" owing to their ease of synthesis and functionalization, relative biocompatibility $^{[41]}$ as well as low toxicity in preliminary assays ${ }^{[9]}$. However, various factors need to be considered in designing an effective drug delivery system. The properties of AuNPs such as their size, charge and surface chemistry have been shown to affect the uptake of AuNPs into cells as well as their subsequent intracellular fate. In addition, effective drug delivery strategies must take into account the nature of drug-AuNP interaction (covalent/non-covalent binding) as well as the means of drug release following introduction of the drug-AuNP complexes to cells ${ }^{[42]}$. If AuNPs are used solely as carriers into cells, it is also critical to monitor any toxic effects of residual materials in the cell after delivery; a biodegradable NP vector whose lifespan is limited to the therapeutic window of the drug would be ideal ${ }^{[43]}$. If the NP vector is cleared from the system once its purpose is reached, it will reduce exposure and limit its toxic effects in the body.

Another issue of concern is the penetration rate of AuNPs into tumors and the specificity of the target sites. Particularly, the epithelial and endothelial barriers are considered to be the main hindrance for the NPs to overcome. Penetration enhancers like metalloproteases against basement membranes and toxins against intracellular tight junctions, may be useful in aiding the uptake of drug-loaded AuNPs into the tumor ${ }^{[44]}$. Another factor to be considered is the AuNP retention in blood circulation. Some researchers have found that particle retention is also size-dependent and longer circulation time is correlated to higher rate of reaching tumor target ${ }^{[45]}$. In addition, most studies have only investigated on drug delivery to solid tumors, where it is site specific and easier for quantification of results. It remains to be seen if AuNPs will be effective against non-solid cancers like leukemia where strategies for targeting and treating such cancers can be different from that for solid tumors.

Drug attachment and release from NPs is another challenging area. While the ease of surface modification is what makes AuNP attractive for drug delivery, the strength of drug attachment and timing of the release needs to be suitably controlled to produce the highest therapeutic efficacy. Foremost, the method of release at the tumor site is dependent on how the drug is attached to the AuNP, whether covalently or through non-covalent binding. Generally, drugs in the active form are loaded non-covalently while the covalent-conjugation of the drug to AuNP is in the pro-drug form, thereby requiring a second reaction to release the drug from the attachment as well as to activate it. Although there have been quite a number of strategies proposed for the triggering of drug release at the tumor site, they can generally be narrowed down to three methods: light or photothermal release ${ }^{[46,47]}$, glutatione-mediated $^{[48]}$, and non-covalent encapsulation of the active drug with subsequent off-loading by diffusion through the membrane $^{[9]}$. The others are principally a modification of one or a combination of these methods. Thus far, the in vitro works done by Kim et $a l^{[9]}$ have yielded promising results. However, more work is required as there is still the need to assess if these methods are practical for application in vivo.

Similarly in the field of cancer therapy, AuNPs are currently being explored as potential drug delivery agents for the introduction of drugs into tumor cells ${ }^{[49]}$. Cells are known to take up colloidal AuNPs of various shapes and sizes ${ }^{[22]}$ either by specific (via ligand-receptor interaction) or non-specific means. An example of AuNPs being taken up by breast cancer cells in vitro is shown in Figure 2. In order to ensure the specific killing of cancer cells while sparing healthy cells, AuNPs were conjugated with appropriate surface ligands which directed them only to tumor cells (Figure 3). Huang et al (2008) have described two methods for tumor targeting: the first involved conjugation of AuNPs to PEG, and the second involved conjugation of AuNPs with specific antibodies which

A
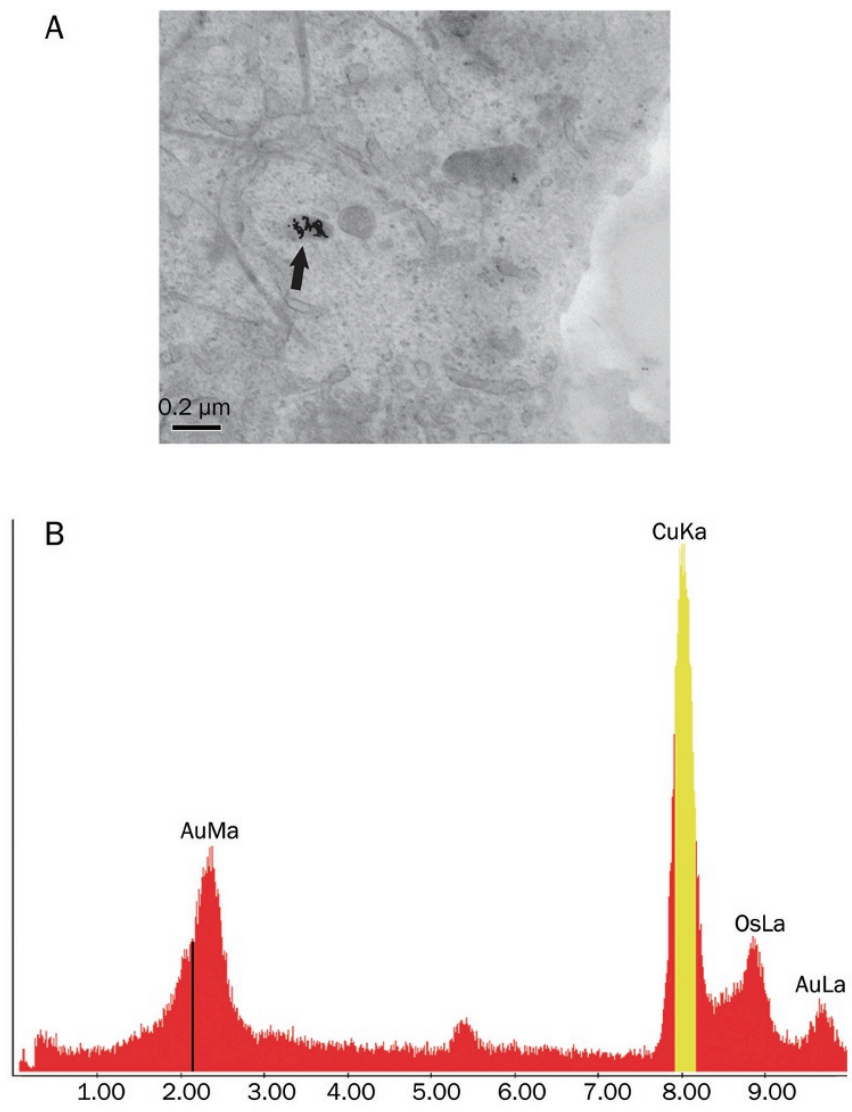

Figure 2. Transmission electron microscopy (TEM) of AuNP treated MCF-7 breast cancer cells. The cells were treated with $1 \mathrm{nmol} / \mathrm{L}$ AuNP for $72 \mathrm{~h}$. (A) A cluster of AuNPs (indicated by an arrow) is found in the cytoplasm of a cell. Bar $=0.2 \mu \mathrm{m}$. (B) TEM specimens were subjected for elemental analysis with a CM120 BioTWIN electron microscope coupled with a Philips EDAX Microanalysis system. The electron dense particles in AuNP treated cells showed the presence of two peaks corresponding to the gold $\mathrm{M}$ shell $(2.2 \mathrm{KeV})$ and $\mathrm{L}$ shell $(9.7 \mathrm{KeV})$. The treatment sample, registered a $P / B$ ratio (ratio of the intensity of the detected element against the background) of 230.27 (Au L shell). For the element to be significantly present in the sample, the $\mathrm{P} / \mathrm{B}$ ratio value needs to be 3.0 and above. 


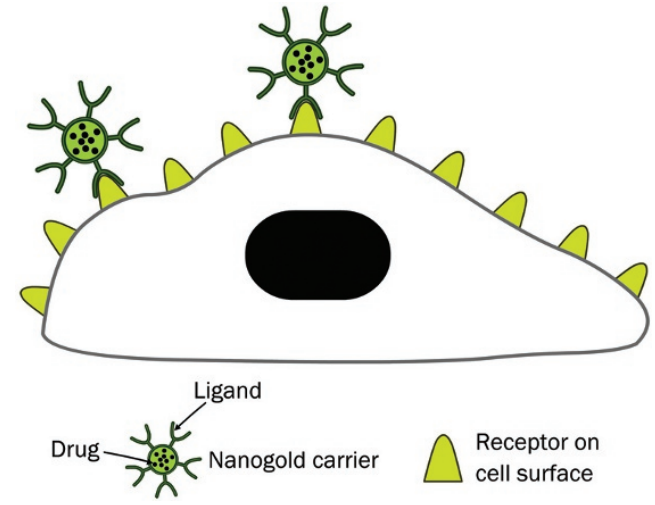

Figure 3. Schematic diagram showing AuNP carriers conjugated with anticancer drugs and ligands which are recognized by receptors on the surface of tumor cells.

bind unique biomarkers expressed on tumor cells ${ }^{[50]}$. PEG prevented AuNP aggregation and lengthened their retention time in blood. This facilitated the preferential accumulation of AuNPs in tumor cells over healthy cells because of the elevated permeability of poorly differentiated blood vessels around tumors following angiogenesis (Figure 4), as well as the decreased clearance rate caused by the deficit of functional lymphatic vessels in tumors ${ }^{[4]}$. Using PEG is considered a passive targeting approach, as opposed to the active targeting of tumor cells through the help of specific antibodies. Following cellular uptake, AuNPs are stored in endosomal/lysosomal vesicles. In order to liberate these AuNPs and introduce the drug which has been delivered into the cell cytoplasm, the NPs need to be modified by the conjugation of membranetranslocating sequence-based peptides which enable them to traverse monolayers ${ }^{[51]}$.

Tumor necrosis factor-alpha (TNF-a) is a potent, multi-
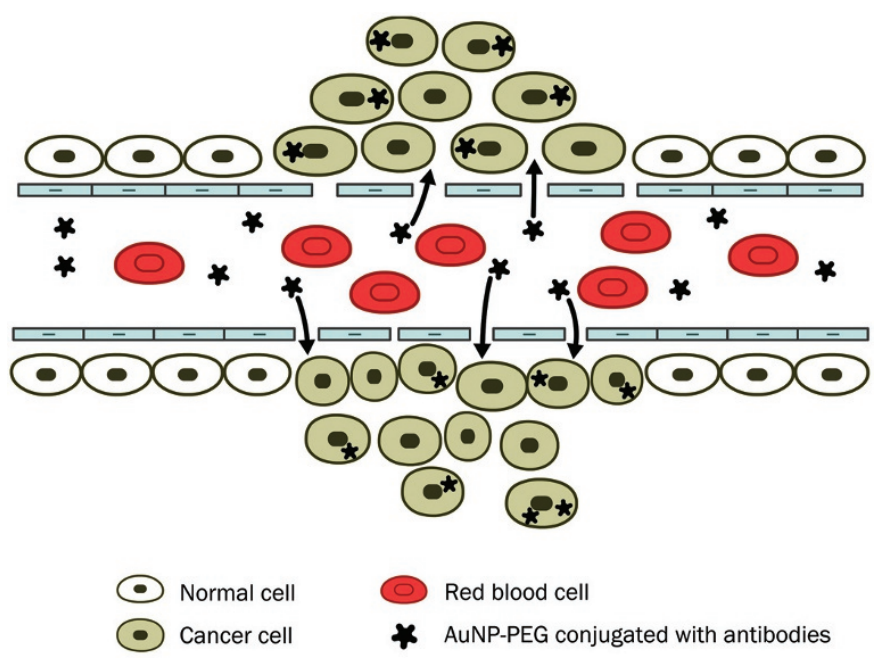

Figure 4. Schematic diagram showing accumulation of ligand-targeted gold nanoparticles conjugated with anticancer drugs in cancer cells mediated via extravasation of the gold nanocarriers through gaps in the endothelial cells (“leaky tumor vasculature”). functional cytokine which not only plays a critical role in inflammation and immunity, but also exhibits anticancer properties $^{[52]}$. However, its systemic toxicity due to their indiscriminate actions on both normal and malignant tissues is well established $^{[16]}$. Indeed, it was shown in one study using the mouse model that increasing doses of native TNF correlated with the severity of toxicities observed ${ }^{[49]}$. In the same study, it was revealed that in contrast to native TNF, TNF conjugated to a colloidal gold platform interspersed with thiol-derivatized PEG was more efficacious in reducing tumor burden in a colon cancer xenograft model, without causing death of the animals.

A Phase 1 clinical trial on the PEGylated colloidal gold-TNF construct (CYT-6091) conducted in patients with advanced stage solid cancers has shown potential ${ }^{[53]}$. The CYT-6091 complex appears to be well-tolerated in this first clinical trial on human subjects although fever developed in two patients, which was not unexpected (as evidenced by preclinical data) and were easily controlled. It is unclear if the fever was due to a reaction to the AuNPs or the recombinant TNF (rhTNF) construct. It appears that the AuNP and rhTNF construct produces less adverse reaction than the rhTNF alone even at the highest drug-AuNP concentrations in the pre-clinical findings. Dose-limiting toxic reaction of hypotension was also not seen in any patient under the trial although there were some retrafficking of leukocytes. AuNPs were found in the tumor as well as in the liver biopsies but no toxic adverse effects were observed. In sum, the authors concluded that the clinical results correlated well with the preclinical data, which bodes well for future translational studies for AuNPs. However, it must be borne in mind that data generated with one type of AuNP may not be extrapolated to other kinds of AuNPs with a different shape, size or surface modification.

In a separate study, the effect of incorporating PEG-coated AuNPs with TNF-a for the targeting killing of SCK mammary carcinomas grown in mice combined with heat treatment has also been investigated ${ }^{[54]}$. While AuNPs loaded with TNF- $\alpha$ alone and heat treatment alone showed tumor growth delay, the most drastic effect was observed when TNF-a loaded AuNPs were intravenously introduced, followed by local heating. This combination treatment proved effective in decreasing the in vivo and in vitro tumor cell survival rates, demonstrating the prospect of using AuNPs as drug delivery carriers coupled with subsequent thermal treatment for effective eradication of tumor cells.

The effects of AuNPs conjugated with methotrexate (MTX) in inducing cytoxicity in vitro and anti-tumorigenic effects in vivo have been reported ${ }^{[55]}$. It was observed that accumulation of Au-MTX in tumor cells occurred more rapidly and at higher concentrations than those treated with free MTX. As a result, enhanced cytotoxic effects were also present in several tumor cell lines compared with an identical dosage of free MTX. These results warrant further investigation as they suggest that the conjugation of AuNPs with a chemotherapeutic drug such as MTX was more efficacious than the administration of free MTX alone, displaying the potential of AuNPs as drug carriers targeting only tumor cells. 
However, given the vast array of AuNPs of different shapes and sizes, it is still unclear which type(s) of AuNPs would be the most suitable for drug delivery applications ${ }^{[39]}$. It is likely that this variety of AuNPs will be custom made to suit the needs for patient treatment. The use of AuNPs in such a manner is not without its disadvantages. There are inherent problems and potential problems to the use of NPs in the delivery of drugs to the target site such as size of the NP and drug conjugate, intratumoral pressure and differential expression of receptors at the tumor site. Optimism towards the utilization of AuNPs as drug delivery vectors into cells should be kept in check as many of the complications regarding targeted drug delivery as well as the toxicity of NPs to cells is a concern yet to be fully addressed. Therefore, the use of AuNPs prior to appropriate assessment of their toxic effects may reap more harm than benefit.

\section{AuNPs in plasmonic photothermal therapy}

Traditionally, heat has been used in the treatment of cancer via the induction of hyperthermia, a condition in which cells are subjected to high temperatures which kill them. While the sources of heat varies from microwaves, radiowaves, ultrasound waves to laser light in the past, such approaches to cancer therapy have not been widely used because of the consequential damage to normal tissues surrounding the targeted tumor. With the advent of nanotechnology, diverse nanostructures have been manufactured for the purpose of photothermal therapeutics. Noble metal NPs such as AuNPs (and including Au nanospheres, nanorods, and nanocages) attract particular interest because they possess enhanced absorption cross-sections $^{[50,56]}$. Their strong absorbance enables effective laser therapy with minimal "collateral damage" to the surrounding healthy tissue. The mechanism by which AuNPs exert their photothermal effect is through SPR. This leads to the formation of a heated electron gas which then cools rapidly within about 1 ps through exchanging energy with the NP lattice. The NP lattice in turn heats up the surrounding environment through the rapid transfer of energy spanning only about $100 \mathrm{ps}^{[57]}$. The speed at which energy is converted and dissipated to the surrounding environment presents an efficient means of rapidly inducing hyperthermia in the vicinity of AuNPs following irradiation with light. Irreversible cell damage resulting from denaturation of proteins and disruption of cell membrane will occur in the areas subjected to high temperatures.

The underlying concept of using antibody-conjugated AuNPs hinges on the necessity of tumor cells in expressing characteristic biomarkers, which are otherwise absent or expressed in significantly lower levels in normal cells. The successful detection and eradication of breast carcinoma cells overexpressing human epidermal growth factor receptor 2 (HER2) through the usage of anti-HER2 immunotargeted gold nanoshells with subsequent irradiation by near infra-red light to potentiate the gold nanoshells-induced photothermal effect has been reported ${ }^{[58]}$. The antibody-mediated targeting of AuNPs to tumor cells was considered to be the more specific and efficient of the two approaches to tumor targeting. It is apparent from here that plasmonic AuNPs exhibit vast potential in the field of photothermal cancer therapy by providing a means to specifically target tumor cells.

\section{AuNPs in radiation therapy}

Numerous studies have revealed that AuNPs may have important applications as radiosensitizers (which are drugs that potentiate the effect of radiation for cancer therapy). A study on mice bearing subcutaneous EMT-6 mammary carcinomas showed that not only were AuNPs (1.9 nm in diameter) non-toxic in nature and cleared from the body via the kidneys, they possessed the ability to enhance the effect of X-ray therapy leading to a remarkable survival rate of $86 \%$ as opposed to $20 \%$ with X-rays alone and $0 \%$ with AuNPs alone ${ }^{[59]}$. However, it is crucial to note that while $1.9 \mathrm{~nm}$ AuNPs seem to show potential as radiation enhancing agents, a recent study discovered evidence for acute cytotoxicity, DNA damage and apoptosis mediated by oxidative stress induced by cellular uptake of $1.9 \mathrm{~nm} \mathrm{AuNPs}{ }^{[60]}$. There is a need for further understanding of cellular responses to AuNPs when exploring their potential to be used in radiation therapy to cure cancer.

The effectiveness of AuNPs as radiosensitizers seems to be closely related to their surface functionality. While the above studies utilized uncoated AuNPs, another study showed that $5 \mathrm{~nm}$ AuNPs coated with the gadolinium chelating agent dithiolated diethylenetriaminepentaacetic gadolinium (Au@ DTDTPA:Gd) did not exhibit radiosensitizing effect in both tumor cells in vitro and in vivo models (MC7-L1 tumor-bearing mice $)^{[61]}$. Instead, a chemotherapeutic effect was observed, which warrants further investigation. The authors suggest that the radiosensitizing properties of AuNPs could possibly be strongly reliant on the nature of their coating. However, the discrepancy over the radiosensitizing effects of AuNPs may also be attributed to the different dimensions of AuNPs used as well as the type of tumor cells under study.

\section{AuNPs as antiangiogenic agents}

Interestingly, AuNPs have been reported to possess antiangiogenic property ${ }^{[62]}$. The exact mechanism of action is still not clearly understood but it was observed that AuNPs bind preferentially to vascular permeability factor/vascular endothelial growth factor (VPF/VEGF)-165 and basic fibroblast growth factor (bFGF) primarily through the heparin-binding domain. This has led researchers to suggest that AuNPs are able to inhibit angiogenesis by preventing the downstream signaling effects of these mitogens on angiogenesis in cancer cells ${ }^{[63]}$.

\section{Conclusion}

The field of NP research presents exciting potential for biomedical applications. Together with an expanding knowledge base on the properties and effects of AuNPs, they are currently explored as potential tools for cancer therapy. Presently, exploiting AuNPs as sensitive probes in the detection and imaging of tumors for diagnostic purposes, delivery agents for the specific targeting of chemotherapeutic drugs to tumor 
cells, and enhancers in plasmonic photothermal therapy and radiation therapy for the eradication of tumor cells appear to show promise. In nanomedicine, the ultimate aim is to utilize NPs efficiently for the in vivo targeted killing of tumor cells with no or minimal side effects. However, even the concept of attaching ligands to the NPs so as to allow them to hone to the tumor appears logical and simple but is in fact fraught with difficulties. In this light, NP research is still at its infancy since many factors remain to be optimized before the application of NPs in cancer therapy becomes a clinical reality.

\section{Acknowledgements}

The authors are grateful to Song-Lin BAY for her assistance in preparing the figures. This work was supported by research funding from the Singapore Ministry of Education Academic Research Fund Tier 1 via grant R279-000-205-112 and Tier 2 via grant MOE2008-T2-1-046, and the National University of Singapore Environmental Research Institute (NERI) via grant R706-000-002-646.

\section{References}

1 Cancer [homepage on the Internet]. Geneva: World Health Organization. Accessed Oct 21, 2010. Available from: http://www.who.int/ cancer/en/.

2 Wagstaff KM, Jans DA. Nuclear drug delivery to target tumour cells. Eur J Pharmacol 2009; 625: 174-80.

3 Boisselier E, Astruc D. Gold nanoparticles in nanomedicine: preparations, imaging, diagnostics, therapies and toxicity. Chem Soc Rev 2009; 38: 1759-82.

4 Liu Y, Miyoshi H, Nakamura M. Nanomedicine for drug delivery and imaging: a promising avenue for cancer therapy and diagnosis using targeted functional nanoparticles. Int J Cancer 2007; 120: 2527-37.

5 Lanone S, Boczkowski J. Biomedical applications and potential health risks of nanomaterials: molecular mechanisms. Curr Mol Med 2006; 6: 651-63.

6 Lewinski N, Colvin V, Drezek R. Cytotoxicity of nanoparticles. Small 2008; 4: 26-49.

7 Jain KK. The role of nanobiotechnology in drug discovery. Adv Exp Med Biol 2009; 655: 37-43.

8 DeLong RK, Reynolds CM, Malcolm Y, Schaeffer A, Severs T, Wanekaya A. Functionalized gold nanoparticles for the binding, stabilization, and delivery of therapeutic DNA, RNA, and other biological macromolecules. Nanotechnol Sci Appl 2010; 2010: 53-63.

9 Kim C, Ghosh P, Rotello V. Multimodal drug delivery using gold nanoparticles. Nanoscale 2009; 1: 61-7.

10 Cobley CM, Chen J, Cho EC, Wang LV, Xia Y. Gold nanostructures: a class of multifunctional materials for biomedical applications. Chem Soc Rev 2011; 40: 44-56.

11 Zrazhevskiy P, Gao X. Multifunctional quantum dots for personalized medicine. Nano Today 2009; 4: 414-28.

12 Biju V, Itoh T, Anas A, Sujith A, Ishikawa M. Semiconductor quantum dots and metal nanoparticles: syntheses, optical properties, and biological applications. Anal Bioanal Chem 2008; 391: 2469-95.

13 Partha R, Conyers JL. Biomedical applications of functionalized fullerene-based nanomaterials. Int J Nanomedicine 2009; 4: $261-$ 75.

14 Sun C, Lee JS, Zhang M. Magnetic nanoparticles in MR imaging and drug delivery. Adv Drug Deliv Rev 2008; 60: 1252-65.

15 Sun C, Veiseh O, Gunn J, Fang C, Hansen S, Lee D, et al. In vivo MRI detection of gliomas by chlorotoxin-conjugated superparamagnetic nanoprobes. Small 2008; 4: 372-9.

16 Powell AC, Paciotti GF, Libutti SK. Colloidal gold: a novel nanoparticle for targeted cancer therapeutics. Methods Mol Biol 2010; 624: 37584.

17 Connor E, Mwamuka J, Gole A, Murphy C, Wyatt M. Gold nanoparticles are taken up by human cells but do not cause acute cytotoxicity. Small 2005; 1: 325-7.

18 Shukla R, Bansal V, Chaudhary M, Basu A, Bhonde RR, Sastry M. Biocompatibility of gold nanoparticles and their endocytotic fate inside the cellular compartment: a microscopic overview. Langmuir 2005; 21: 10644-54.

19 Pan Y, Neuss S, Leifert A, Fischler M, Wen F, Simon U, et al. Sizedependent cytotoxicity of gold nanoparticles. Small 2007; 3: 1941-9.

20 Chen YS, Hung YC, Liau I, Huang GS. Assessment of the in vivo toxicity of gold nanoparticles. Nanoscale Res Lett 2009; 4: 858-64.

21 Johnston H, Hutchison G, Christensen F, Peters S, Hankin S, Stone V. A review of the in vivo and in vitro toxicity of silver and gold particulates: particle attributes and biological mechanisms responsible for the observed toxicity. Crit Rev Toxicol 2010; 40: 328-46.

22 Chithrani B, Ghazani A, Chan W. Determining the size and shape dependence of gold nanoparticle uptake into mammalian cells. Nano Lett 2006; 6: 662-8.

23 Pan Y, Leifert A, Ruau D, Neuss S, Bornemann J, Schmid G, et al. Gold nanoparticles of diameter $1.4 \mathrm{~nm}$ trigger necrosis by oxidative stress and mitochondrial damage. Small 2009; 5: 2067-76.

24 Li J, Zou L, Hartono D, Ong C, Bay B, Yung L. Gold nanoparticles induce oxidative damage in lung fibroblasts in vitro. Adv Mater 2008; 20: $138-42$.

25 Li J, Hartono D, Ong C, Bay B, Yung L. Autophagy and oxidative stress associated with gold nanoparticles. Biomaterials 2010; 31: 59966003.

26 Tedesco S, Doyle H, Blasco J, Redmond G, Sheehan D. Oxidative stress and toxicity of gold nanoparticles in Mytilus edulis. Aquat Toxicol 2010; 100: 178-86.

27 Browning L, Lee K, Huang T, Nallathamby P, Lowman J, Xu X. Random walk of single gold nanoparticles in zebrafish embryos leading to stochastic toxic effects on embryonic developments. Nanoscale 2009; 1: 138-52.

28 De Jong W, Hagens W, Krystek P, Burger M, Sips A, Geertsma R. Particle size-dependent organ distribution of gold nanoparticles after intravenous administration. Biomaterials 2008; 29: 1912-9.

29 Sonavane G, Tomoda K, Makino K. Biodistribution of colloidal gold nanoparticles after intravenous administration: effect of particle size. Colloids Surf B Biointerfaces 2008; 66: 274-80.

30 Cho W, Cho M, Jeong J, Choi M, Cho H, Han B, et al. Acute toxicity and pharmacokinetics of $13 \mathrm{~nm}$-sized PEG-coated gold nanoparticles. Toxicol Appl Pharmacol 2009; 236: 16-24.

31 Cho W, Kim S, Han B, Son W, Jeong J. Comparison of gene expression profiles in mice liver following intravenous injection of 4 and 100 nm-sized PEG-coated gold nanoparticles. Toxicol Lett 2009; 191: 96-102.

32 Kumar S, Harrison N, Richards-Kortum R, Sokolov K. Plasmonic nanosensors for imaging intracellular biomarkers in live cells. Nano Lett 2007; 7: 1338-43.

33 Sperling R, Rivera Gil P, Zhang F, Zanella M, Parak W. Biological applications of gold nanoparticles. Chem Soc Rev 2008; 37: 1896908.

34 Roth J. The silver anniversary of gold: 25 years of the colloidal gold marker system for immunocytochemistry and histochemistry. Histochem Cell Biol 1996; 106: 1-8. 
35 Lee S, Chon H, Lee M, Choo J, Shin S, Lee Y, et al. Surface-enhanced Raman scattering imaging of HER2 cancer markers overexpressed in single MCF7 cells using antibody conjugated hollow gold nanospheres. Biosens Bioelectron 2009; 24: 2260-3.

36 Kneipp J, Kneipp H, Wittig B, Kneipp K. Novel optical nanosensors for probing and imaging live cells. Nanomedicine 2010; 6: 214-26.

37 Kneipp K, Kneipp H, Kneipp J. Surface-enhanced Raman scattering in local optical fields of silver and gold nanoaggregates-from singlemolecule Raman spectroscopy to ultrasensitive probing in live cells. Acc Chem Res 2006; 39: 443-50.

38 Qian X, Peng X, Ansari D, Yin-Goen Q, Chen G, Shin D, et al. In vivo tumor targeting and spectroscopic detection with surface-enhanced Raman nanoparticle tags. Nat Biotechnol 2008; 26: 83-90.

39 Cai W, Gao T, Hong H, Sun J. Applications of gold nanoparticles in cancer nanotechnology. Nanotechnol Sci Appl 2008; 1: 17-32.

40 de la Escosura-Muñiz A, Sánchez-Espinel C, Díaz-Freitas B, GonzálezFernández A, Maltez-da Costa M, Merkoçi A. Rapid identification and quantification of tumor cells using an electrocatalytic method based on gold nanoparticles. Anal Chem 2009; 81: 10268-74.

41 Lee SH, Bae KH, Kim SH, Lee KR, Park TG. Amine-functionalized gold nanoparticles as non-cytotoxic and efficient intracellular siRNA delivery carriers. Int J Pharm 2008; 364: 94-101.

42 Duncan B, Kim C, Rotello VM. Gold nanoparticle platforms as drug and biomacromolecule delivery systems. J Control Release 2010; 148: $122-7$.

43 De Jong W, Borm P. Drug delivery and nanoparticles: applications and hazards. Int J Nanomedicine 2008; 3: 133-49.

44 Sakamoto J, Annapragada A, Decuzzi P, Ferrari M. Antibiological barrier nanovector technology for cancer applications. Expert Opin Drug Deliv 2007; 4: 359-69.

45 Zhang G, Yang Z, Lu W, Zhang R, Huang Q, Tian M, et al. Influence of anchoring ligands and particle size on the colloidal stability and in vivo biodistribution of polyethylene glycol-coated gold nanoparticles in tumor-xenografted mice. Biomaterials 2009; 30: 1928-36.

46 Bikram M, Gobin AM, Whitmire RE, West JL. Temperature-sensitive hydrogels with $\mathrm{SiO}_{2}$-Au nanoshells for controlled drug delivery. J Control Release 2007; 123: 219-27.

47 Agasti SS, Chompoosor A, You CC, Ghosh P, Kim CK, Rotello VM. Photoregulated release of caged anticancer drugs from gold nanoparticles. J Am Chem Soc 2009; 131: 5728-9.

48 Hong R, Han G, Fernandez JM, Kim BJ, Forbes NS, Rotello VM. Glutathione-mediated delivery and release using monolayer protected nanoparticle carriers. J Am Chem Soc 2006; 128: 1078-9.

49 Paciotti GF, Myer L, Weinreich D, Goia D, Pavel N, McLaughlin RE, et al. Colloidal gold: a novel nanoparticle vector for tumor directed drug delivery. Drug Deliv 2004; 11: 169-83.

50 Huang X, Jain P, El-Sayed I, El-Sayed M. Plasmonic photothermal therapy (PPTT) using gold nanoparticles. Lasers Med Sci 2008; 23 : 217-28.

51 Koch A, Reynolds F, Merkle H, Weissleder R, Josephson L. Transport of surface-modified nanoparticles through cell monolayers. Chembiochem 2005; 6: 337-45.

52 van Horssen R, Ten Hagen T, Eggermont A. TNF-alpha in cancer treatment: molecular insights, antitumor effects, and clinical utility. Oncologist 2006; 11: 397-408.

53 Libutti SK, Paciotti GF, Byrnes AA, Alexander HR Jr, Gannon WE, Walker M, et al. Phase I and pharmacokinetic studies of CYT-6091, a novel PEGylated colloidal gold-rhTNF nanomedicine. Clin Cancer Res 2010; 16: 6139-49.

54 Visaria R, Griffin R, Williams B, Ebbini E, Paciotti G, Song C, et al. Enhancement of tumor thermal therapy using gold nanoparticleassisted tumor necrosis factor-alpha delivery. Mol Cancer Ther 2006; 5: 1014-20.

55 Chen Y, Tsai C, Huang P, Chang M, Cheng P, Chou C, et al. Methotrexate conjugated to gold nanoparticles inhibits tumor growth in a syngeneic lung tumor model. Mol Pharm 2007; 4: 713-22.

56 Liu C, Li BQ, Mi CC. Fast transient thermal analysis of gold nanoparticles in tissue-like medium. IEEE Trans Nanobioscience 2009; 8: 271-80.

57 Link S, El-Sayed MA. Shape and size dependence of radiative, nonradiative and photothermal properties of gold nanocrystals. Int Rev Phys Chem 2000; 19: 409-53.

58 Loo C, Lowery A, Halas N, West J, Drezek R. Immunotargeted nanoshells for integrated cancer imaging and therapy. Nano Lett 2005; 5: 709-11.

59 Hainfeld J, Slatkin D, Smilowitz $H$. The use of gold nanoparticles to enhance radiotherapy in mice. Phys Med Biol 2004; 49: N309-15.

60 Butterworth KT, Coulter JA, Jain S, Forker J, McMahon SJ, Schettino $\mathrm{G}$, et al. Evaluation of cytotoxicity and radiation enhancement using $1.9 \mathrm{~nm}$ gold particles: potential application for cancer therapy. Nanotechnology 2010; 21: 295101.

61 Hébert E, Debouttière P, Lepage M, Sanche L, Hunting D. Preferential tumour accumulation of gold nanoparticles, visualised by magnetic resonance imaging: Radiosensitisation studies in vivo and in vitro. Int J Radiat Biol 2010; 86: 692-700.

62 Mukherjee P, Bhattacharya R, Wang P, Wang L, Basu S, Nagy JA, et al. Antiangiogenic properties of gold nanoparticles. Clin Cancer Res 2005; 11: 3530-4.

63 Bhattacharya R, Mukherjee P. Biological properties of "naked" metal nanoparticles. Adv Drug Deliv Rev 2008; 60: 1289-306. 